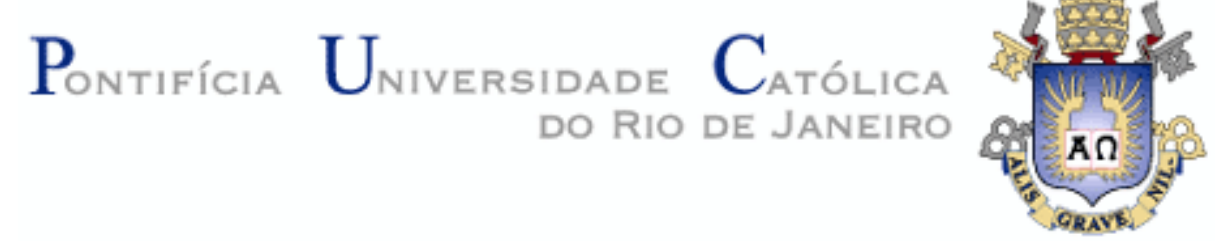

Michel Nunes Lopes Masson

\title{
No Fio da Borda: \\ O Conceitualismo de Dan Graham
}

Tese apresentada ao Programa de Pós-Graduação em História Social da Cultura do Departamento de História da PUC-Rio como parte dos requisitos parciais para obtenção do grau de Doutor em História.

Orientador: Prof. Ronaldo Brito Fernandes 


\section{Michel Nunes Lopes Masson \\ No Fio da Borda: O Conceitualismo de Dan Graham}

Tese apresentada como requisito parcial para obtenção do grau de Doutor pelo Programa de Pós-Graduação em História Social da Cultura do Departamento de História do Centro de Ciências Sociais da PUC-Rio. Aprovada pela Comissão Examinadora abaixo assinada.

Prof ${ }^{\circ}$ Ronaldo Brito Fernandes Orientador

Departamento de História - PUC-Rio

Prof $f^{a}$ Eleonora Batista Fabião Escola de Comunicação - UFRJ

Prof ${ }^{a}$ Patricia Leal Azevedo Corrêa Escola de Belas Artes - UFRJ

Prof ${ }^{a}$ Vera Beatriz Cordeiro Siqueira Instituto de Artes - UERJ

Profo Ricardo Roclaw Basbaum Instituto de Artes - UERJ

Prof ${ }^{\text {a }}$ Mônica Herz Vice-Decana de Pós-Graduação do Centro de Ciências Sociais PUC-Rio

Rio de Janeiro, 10 de dezembro de 2014 
Todos os direitos reservados. É proibida a reprodução total ou parcial do trabalho sem autorização da universidade, do autor e do orientador.

\section{Michel Nunes Lopes Masson}

Graduou-se em Arquitetura e Urbanismo pela Faculdade de Arquitetura e Urbanismo da UFRJ em 1999. Obteve Especialização em História da Arte e da Arquitetura do Brasil pela PUC-Rio em 2005. Concluiu o mestrado em Design pela PUC-Rio em 2003. Tem experiência nas áreas de História e Teoria da Arte, com ênfase em arte moderna e contemporânea.

Ficha Catalográfica

Masson, Michel Nunes Lopes

No fio da borda: o conceitualismo de Dan Ghaham / Michel Nunes Lopes Masson ; orientador: Ronaldo Brito Fernandes. - 2015.

233 f. : il. (color.) ; $30 \mathrm{~cm}$

Tese (doutorado)-Pontifícia Universidade Católica do Rio de Janeiro, Departamento de História, 2015.

Inclui bibliografia

1. História - Teses. 2. História social da cultura. 3. Graham, Dan. 4. Arte contemporânea norte americana. 5. Pós-minimalismo. 6. Conceitualismo. 7. Cultura. 8. Arquitetura. I. Fernandes, Ronaldo Brito. II. Pontifícia Universidade Católica do Rio de Janeiro. Departamento de História. III. Título. 


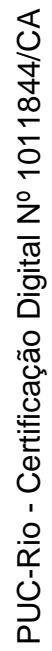

Para meus pais, Eliane e Paulo.

Para vocês tudo, sempre. 


\section{Agradecimentos}

A meu orientador, professor Ronaldo Brito, pelo privilégio das orientações dedicadas e sempre precisas.

À Capes, à PUC-Rio e à Columbia University, pelos auxílios concedidos.

À Graduate School of Architecture, Planing and Preservation, pela oportunidade de pesquisa na Avery Library.

Aos professores John Miller, da Barnard College, e Mark Wigley, da GSAPP, que gentilmente permitiram que eu assistisse a seus cursos.

A Cecilia Cotrim de Mello que, em meio a um momento inicial de hesitação de minha parte, me incentivou e me ajudou a seguir na direção de meu tema.

Aos funcionários do Departamento de História da PUC-Rio, Anair dos Santos, Cleusa Ventura, Claudio Santiago e, especialmente, Edna Timbó, pela presteza e paciência.

Aos professores do Departamento de História da PUC-Rio, em especial Marcelo Gantus Jasmin e João Masao Kamita.

Aos integrantes da banca examinadora, Eleonora Fabião, Vera Beatriz Siqueira, Patricia Correia e Ricardo Basbaum, pela competência e lucidez de seus comentários.

A Ana Luiza Nobre, pela contribuição na escolha dos nomes da banca.

A meu amigo Pedro Segreto, que me disponibilizou toda a estrutura de seu escritório para digitalizar as imagens desta tese.

Ao querido Otavio Schipper, pelas conversas sobre topologia e arte.

A meu pai, Paulo Masson, pelo interesse e atenção; suas palavras são sempre um acalanto nos momentos difíceis.

A minha mãe, Eliane Lopes, por seu apoio incondicional nesse caminho. A meus irmãos, Aline Lopes e Bernardo Paim.

A Maria Helena Torres, pela dedicação e boa vontade na revisão deste texto.

Especialmente a Marianne Antabi, minha companheira, que, com seu expediente e apoio incondicional, leu o texto, digitalizou e tratou as imagens, diagramou este estudo junto comigo, além de editar o vídeo para a defesa da tese, por ela gravada. Jamais esquecerei tamanho exemplo de bondade e cumplicidade. 


\section{Resumo}

Masson, Michel Nunes Lopes; Fernandes, Ronaldo Brito (Orientador). No Fio da Borda: O Conceitualismo de Dan Graham. Rio de Janeiro, 2014, 233p. Tese de Doutorado - Departamento de História, Pontifícia Universidade Católica do Rio de Janeiro.

Figura-chave da chamada "arte conceitual", o artista norte-americano Dan Graham iniciou sua trajetória artística em meados da década de 1960 muito por acaso. Convidado por amigos, tornou-se sócio da John Daniels, galeria em Nova York bastante ativa, mas de vida breve. Falido, viu-se obrigado a retornar à casa de seus pais em Nova Jersey. Durante a viagem de trem, pôde observar a "nova cidade". Dessa experiência surgiu Homes for America, notório ensaio fotográfico sobre as habitações em massa dos subúrbios norte-americanos, que integra um primeiro conjunto de trabalhos do artista destinados a revistas. Algum tempo depois, Graham ingressou na Nova Scotia College of Art and Design, Halifax, dando início a um período em que seu interesse se volta para o vídeo, a performance e a instalação, interrompido por Public Space/Two Audiences, trabalho realizado para a Bienal de Veneza de 1976 que impõe ao artista uma autocrítica. A despeito de seu êxito, Graham reconhece que a instalação se aproximara em demasia do conceito de "cubo branco". Como saída para o impasse, adotou a ideia de "plano da cidade", passando a propor projetos de intervenção urbana até iniciar, no começo dos anos 80, seus pavilhões. Levando em conta a obra de Graham, ampla e diversa em seus meios, a tese é monografia que tem por objetivo realizar um estudo analítico sobre a prática do artista, definida, a meu ver, nos termos de um modelo crítico-conceitualista.

\section{Palavras-chave}

Dan Graham; arte contemporânea norte-americana; pós-minimalismo; conceitualismo; cultura; arquitetura. 


\section{Abstract}

Masson, Michel Nunes Lopes; Fernandes, Ronaldo Brito (Advisor). On the Edge: The Conceptualism of Dan Graham. Rio de Janeiro, 2014, 233p. Thesis - Departamento de História, Pontifícia Universidade Católica do Rio de Janeiro.

Key figure of the so-called "conceptual art", the North American artist Dan Graham began his artistic career in the mid-1960s quite by chance. Invited by friends, he became a partner of the John Daniels, a gallery in New York very active, but short-lived. Bankrupt, he was forced to return to his parents' home in New Jersey. During the train ride, Graham can saw the "new city". From this experience came Homes for America, notorious photographic essay on mass tract housing of North American suburbs, which is part of a first set of works for magazines. A while later, Graham joined the Nova Scotia College of Art and Design, Halifax, starting a period in which your interest turns to video, performance and installation, interrupted by Public Space/Two Audiences, work done for the Venice Biennale in 1976 that impose the artist self-criticism. Despite its success, Graham recognizes that the installation was near of the concept of the "white cube". As out of the impasse, he adopts the idea of "city plan", going to propose urban interventions projects, starting in the early 80 s their pavilions. Considering Graham's work, broad and diverse in its mediums, the thesis is a monograph that aims to carry out an analytical study of the practice of the artist, defined, in our view, in terms of a critical-conceptualist model.

\section{Keywords}

Dan Graham; North American contemporary art; post minimalism; conceptualism; culture; architecture. 


\section{Sumário}

1. Introdução 13

2. Processualidade e Significado 30

3. Sistemas de Captura $\quad 61$

4. Corpo e Vidro 119

5. Arte e Arquitetura 165

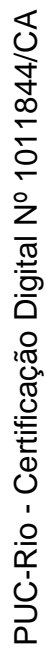

6. Conclusão 210

$\begin{array}{lr}\text { 7. Referências Bibliográficas } & 217\end{array}$ 


\section{Lista de figuras}

Figura 1 - Dan Graham, Bisected Triangle, Interior Curve, 2002, Inhotim, Minas Gerais, Brasil

Figura 2 - Abertura exposição 4D, 1965, Dan Graham (centro), John Daniels Gallery, NY

Figura 3 - Vista exposição Christmas, 1964-65, John Daniels Gallery, NY

Figura 4 - Cartaz Plastics, John Daniels Gallery, NY, 1965

Figura 5 - Donald Judd, Untitled, 1965

Figura 6 - Sol LeWitt, Incomplete Open Cubes 6-1, 9-4, 8-10, 8-19, 8-22, 10-1, 1974

Figura 7 - Dan Graham, Homes for America, layout para artigo de revista, 1972

Figura 8 - Dan Graham, Row of New Tract Houses, Jersey City, Nova Jersey, 1966

Figura 9 - Dan Graham, Courtyard, New Development, Jersey City, Nova Jersey, 1966

Figura 10 - Dan Graham, Split Level 'Two-Home Home', Jersey City, Nova Jersey, 1966

Figura 11 - Dan Graham, Two Family Units, Staten Island, NY, 1967

Figura 12 - Dan Graham, Mother and Son in Backyard, Bayonne, Nova Jersey, 1969

Figura 13 - Dan Graham, Untitled (Family at New High Restaurant), Jersey City, Nova Jersey, 1967

Figura 14 - Dan Graham, Roll of Tract Houses, Bayonne,

Nova Jersey, 1966

Figura 15 - Donald Judd, Concrete Work's, 1980-84,

Chinati Foundation, Marfa

Figura 16 - John Chamberlain, Hanging Herm, 1974

Figura 17 - Jasper Johns, Target with Four Faces, 1955

Figura 18 - Jasper Johns, White Numbers, 1958

Figura 19 - Dan Graham, Scheme, 1965

Figura 20 - Dan Graham, Scheme (book version), 1965/73 48 
Figura 21 - Dan Graham, March 31, 1966, 1966

Figura 22 - Dan Graham, Figurative by Dan Graham, Harper's Bazaar, 1968 50

Figura 23 - Dan Graham, Detumescence, 1966 51

Figura 24 - Dan Graham, Side Effects/Common Drugs, 1966 53

Figura 25 - Dan Graham, Schema (March, 1966), Art-Language v. 1, n. 1,1969

54

Figura 26 - Dan Graham, variante de Schema (March 1966), 1966 56

Figura 27 - Cildo Meireles, Inserções em Circuitos Ideológicos:

Projeto Coca-Cola, 1970

Figura 28 - Dan Graham, Project for a Local Cable TV, 1971,

NSCAD, Halifax, Nova Scotia, Canadá

59

Figura 29 - Capa da revista Radical Software, v. 1, n. 1, 1970

Figura 30 - Steve Reich, Pendulum Music, 1968. Performance

no Whitney Museum of American Art

Figura 31 - Dan Graham, Lax/Relax, 1969. Segunda performance, 1969, NSCAD

Figura 32 - Dan Graham, Two Consciousness Projection(s), 1972.

NSCAD, 1972

Figura 33 - Dan Graham, Past Future Split Attention, 1972

Figura 34 - Dan Graham, Intention Intentionality Sequence,1972,

Protech-Rivkin Gallery, Washignton, D.C., 1972

Figura 35 - Dan Graham, Performer/Audience/Mirror, 1977

Figura 36 - Bruce Nauman, stills de Walking in an Exaggerated

Manner around the Perimeter of a Square, 1968

Figura 37 - Dan Graham, Vanish Point, 1969

Figura 38 - Dan Graham, Sunset to Sunrise, 1969

Figura 39 - Dan Graham, Two Correlated Rotations, 1969

Figura 40 - Dan Graham, Roll, 1970

Figura 41 - Dan Graham, Body Press, 1970-72

Figura 42 - Dan Graham, TV Camera/Monitor Performance, $1970 \quad 93$

Figura 43 - Bruce Nauman, Walk with Contrapposto, 1669

Figura 44 - Bruce Nauman, Performance Corridor, 1969

Figura 45 - Bruce Nauman, Live-Taped Video Corridor, 1970

Figura 46 - Bruce Nauman, Going Around the Corner, 1970 
Figura 47 - Dan Graham, Present Continuous Past(s), 1974

Figura 48 - Dan Graham, Two Rooms/Reverse Video Delay, 1974103

Figura 49 - Dan Graham, Opposing Mirrors and Video Monitors on

Time Delay, 1974

Figura 50 - Dan Graham, Opposing Mirrors and Video Monitors on

Time Delay, 1974

Figura 51 - Dan Graham, Present Continuous Past(s), 1974

Figura 52 - Dan Graham, Public Space/Two Audiences, 1976

Figura 53 - Dan Graham, Video Piece for Showcase Windows in Shopping Arcade, 1976

Figura 54 - Dan Graham, Public Space/Two Audiences, 1976

Figura 55 - Dan Graham, Public Space/Two Audiences, 1976

Figura 56 - Dan Flavin, Untitlet (to Bob and Pat Rohm), 1969

Figura 57 - Joseph Kosuth, Glass Words Material Described, 1965130

Figura 58 - Marc-Antoine Laugier, frontispício de Um ensaio sobre arquitetura, 1753

Figura 59 - Jacques Rigaud, vista do Teatro da Rainha a partir da Rotunda em Stowe, Buckinghamshire, Reino Unido, c. 1739 Figura 60 - Mies van der Rohe, Pavilhão Alemão para Exposição Internacional de Barcelona, 1929

Figura 61 - Dan Graham, Pavilion/Sculpture II, 1984, Estocolmo,

Suécia

Figura 62 - Dan Graham, Half Square/Half Crazy, 2004, Como, Itália 138

Figura 63 - Dan Graham, Half Square/Half Crazy, 2004, Como, Itália

Figura 64 - Pavilhão Amalienburg, Palácio Nymphenburg, Munique, Alemanha, hall de espelhos, Johann Baptist Zimmermann e Joachim Dietrich, 1734-39

Figura 65 - Mies van der Rohe, Seagram Building, Nova York, 1954-58

Figura 66 - Dan Graham, "Reflective-Glass" Office Building,

Los Angeles, 1978 (fotografia)

Figura 67 - Dan Graham, Chill Out Pavillion, Krabbesholm,

Dinamarca, 2010

Figura 68 - Dan Graham, Pavilion/Sculpture for Argone, Chicago, EUA, 1978-81 
Figura 69 - Dan Graham, Bisected Triangle, Interior Curve, Inhotim, Minas Gerais, Brasil, 2002

Figura 70 - Dan Graham, Two Adjacent Pavilions, 1978-82,

Documenta 7, Kassel, Alemanha

153

Figura 71 - Robert Morris, Mirrored Cubes, 1965

153

Figura 72 - Dan Graham, Cinema, 1981

155

Figura 73 - Dan Graham, Double Exposure, 2003, Porto, Portugal

Figura 74 - Dan Graham, Two-Way Mirror Cylinder Inside Cube and Video Lounge, 1986-91, DIA Foundation, NY

Figura 75 - Dan Graham, stills de Rock my Religion, 1982-84

Figura 76 - Dan Graham, stills de Rock my Religion, 1982-84

Figura 77 - Dan Graham, Fun House for Münster, 1997, Roterdã, Holanda

Figura 78 - Dan Graham, Clinic for a Suburban Site, 1978

Figura 79 - Dan Graham, Alteration of a Suburban House, 1978

Figura 80 - Mies van der Rohe, Farnsworth House, 1945-51

Figura 81 - Dan Graham, Video Projection Outside Home, 1978

Figura 82 - Robert Venturi, Guild House, 1960-63

Figura 83 - Dan Graham, Video View of Suburbia in an Urban Atrium, 1979-80

Figura 84 - Kevin Roche, Ford Foundation Building, Nova York, EUA, 1963-67

Figura 85 - John Portman, Hyatt Regency Hotel, Atlanta, EUA, 1967

Figura 86 - Gordon Matta-Clark, Dumpster Duplex,1972

Figura 87 - Gordon Matta-Clark, Bingo, 1974

Figura 88 - Gordon Matta-Clark, Splitting, 1974

Figura 89 - Gordon Matta-Clark, Splitting, 1974, fotocolagem em preto e branco

Figura 90 - Dan Graham, Two Correlated Rotations, 1969

Figura 91 - Dan Graham, Three Linked Cubes/Interior Design for Space Showing Videos, 1986, Rennes, França 